\title{
Thomas-Fermi Model in the Presence of Natural Cutoffs
}

\author{
Kourosh Nozari, Z. Haghani, and J. Vahedi \\ Department of Physics, Islamic Azad University, Sari Branch, Sari, Iran \\ Correspondence should be addressed to Kourosh Nozari; knozari@umz.ac.ir \\ Received 5 November 2013; Revised 26 January 2014; Accepted 29 January 2014; Published 28 April 2014
}

Academic Editor: Elias C. Vagenas

Copyright (C) 2014 Kourosh Nozari et al. This is an open access article distributed under the Creative Commons Attribution License, which permits unrestricted use, distribution, and reproduction in any medium, provided the original work is properly cited. The publication of this article was funded by $\mathrm{SCOAP}^{3}$.

\begin{abstract}
It has been revealed, in the context of quantum gravity candidates, that measurement of position cannot be done with arbitrary precision and there is a finite resolution of space-time points. This leads naturally to a minimal measurable length of the order of Planck length. Also, in the context of newly proposed doubly special relativity theories, a test particle's momentum cannot be arbitrarily imprecise leading nontrivially to a maximal momentum for a test particle. These two natural cutoffs affects most of quantum field theoretic arguments in the spirit of condensed matter physics. Here we focus on the role of these natural cutoffs on Thomas-Fermi theory in condensed matter physics. We show how quantum gravity effects can play important role phenomenologically in many-body interactions of solids.
\end{abstract}

\section{Introduction}

According to equivalence principal in general relativity, gravitational field is coupled to everything. It has been characterized that gravity in very small length scales causes serious change in the structure of space-time. It causes minimal uncertainty in positions of atomic and subatomic particles [115]. In fact, there is an absolutely small uncertainty in position measurement of any quantum mechanical system and this feature leads nontrivially to the existence of a minimal measurable length on the order of Planck length. Existence of this natural cutoff requires deformation of the standard Heisenberg uncertainty principle to the so-called generalized uncertainty principle (GUP) (see, for instance, [13, 14, 16-21]). In one dimension for positions and momentum operators, the deformed Heisenberg algebra can be represented as

$$
[X, P]=i \hbar\left(1+\beta P^{2}\right) \text {. }
$$

In general, for two symmetric operators $A$ and $B$, we have

$$
\Delta A \Delta B \geq|\langle[A, B]\rangle| .
$$

So the generalized uncertainty principle can be deduced as

$$
\Delta X \Delta P \geq \frac{\hbar}{2}\left[1+\beta(\Delta P)^{2}\right] .
$$

While in ordinary quantum mechanics $\Delta X$ can be made arbitrarily small by letting $\Delta P$ grow correspondingly, this is no longer the case if (3) holds. If, for decreasing $\Delta X, \Delta P$ increases, the new term $\beta(\Delta P)^{2}$ on the right hand side of (3) will eventually grow faster than the left hand side. Hence $\Delta X$ can no longer be made arbitrarily small [16-20]. To obtain this minimal uncertainty, we saturate inequality in (3) and solve the resulting equation for $\Delta P$ :

$$
\Delta P=\frac{\Delta X \pm \sqrt{(\Delta X)^{2}-\hbar^{2} \beta}}{\hbar \beta} .
$$

The reality of solutions requires positivity of the term in square root, leading to

$$
(\Delta X)_{0}=\hbar \sqrt{\beta} .
$$

This smallest uncertainty in position measurement leads nontrivially to the existence of a minimal measurable length. On the other hand, in the context of the doubly special relativity (DSR) theories (for review see [22-27]), one can show that a test particle's momentum cannot be arbitrarily imprecise. In fact there is an upper bound for momentum fluctuations [28-31]. As a nontrivial assumption, this may lead to a maximal measurable momentum for a test particle 
(see $[20,32-34])$. In this framework, the GUP that predicts both a minimal observable length and a maximal momentum can be written (with $\hbar=1$ ) as follows [32, 33]:

$$
\begin{aligned}
& \Delta X \Delta P \\
& \geq \frac{1}{2}\left[1+\left(\frac{\alpha}{\sqrt{\left\langle P^{2}\right\rangle}}+4 \alpha^{2}\right)(\Delta P)^{2}+4 \alpha^{2}\langle P\rangle^{2}-2 \alpha \sqrt{\left\langle P^{2}\right\rangle}\right] .
\end{aligned}
$$

Since $(\Delta P)^{2}=\left\langle P^{2}\right\rangle-\langle P\rangle^{2}$, by setting $\langle P\rangle=0$ for simplicity, we find

$$
\Delta X \Delta P \geq \frac{1}{2}\left[1-\alpha(\Delta P)+4 \alpha^{2}(\Delta P)^{2}\right] .
$$

This GUP contains both a minimal length and a maximal momentum. To see how a maximal momentum arises in this setup (see [20], for details), we note that with GUP (7) the absolute minimal measurable length is given by $\Delta X_{\min }(\langle P\rangle=$ $0) \equiv \Delta X_{0}=3 \alpha / 2$. Due to duality of position and momentum operators, it is reasonable to assume $\Delta X_{\min } \propto \Delta P_{\max }$. By saturating the inequality in relation (7), we find

$$
2(\Delta X \Delta P)=\left(1-\alpha(\Delta P)+4 \alpha^{2}(\Delta P)^{2}\right) .
$$

This results in

$$
(\Delta P)^{2}-\frac{(2 \Delta X+\alpha)}{4 \alpha^{2}} \Delta P+\frac{1}{4 \alpha^{2}}=0 .
$$

So we obtain

$$
\left(\Delta P_{\max }\right)^{2}-\frac{\left(2 \Delta X_{\min }+\alpha\right)}{4 \alpha^{2}} \Delta P_{\max }+\frac{1}{4 \alpha^{2}}=0 .
$$

Now by using the value of $\Delta X_{\min }$, we find

$$
\left(\Delta P_{\max }\right)^{2}-\frac{1}{\alpha} \Delta P_{\max }+\frac{1}{4 \alpha^{2}}=0 .
$$

The solution of this equation is

$$
\Delta P_{\max }=\frac{1}{2 \alpha}
$$

So, there is an upper bound on particle's momentum uncertainty. As a nontrivial assumption, we assume that this maximal uncertainty in particle's momentum is indeed the maximal measurable momentum. This is of the order of Planck momentum.

The presence of these strong natural constraints in measurement of position and momentum of particles has been ignored in studies of many-body interactions of condensed matter physics. Based on these preliminaries as our main motivation, we are going to study Thomas-Fermi theory in the presence of these natural cutoffs.

Thomas-Fermi theory is a basic theory in many-body interactions. For a system that consists of $N$ particles each with mass $m$, the deformed algebra (1) is as follows:

$$
\left[X_{j}, P_{k}\right]=i \hbar \delta_{j k}\left(1+\beta P_{k}^{2}\right), \quad\left[X_{j}, X_{k}\right]=\left[P_{j}, P_{k}\right]=0,
$$

where $j, k=1, \ldots, N$. If the particles of system are interacting via the pairwise potential $V$, the Hamiltonian of our $N$-body system is given by [24]

$$
H^{N}=\sum_{j=1}^{N} \frac{P_{j}^{2}}{2 m}+\sum_{j<k=1}^{N} V\left(X_{j}-X_{k}\right) .
$$

Generally, Hamiltonian of a many-body system with variety of interactions between particles is too difficult to handle. Nevertheless, some progress can be made if we neglect mutual interactions of particles. In this first step analysis the total Hamiltonian of the many-body system transforms to a set of independent single-particle Hamiltonians and these are usually easy to solve. A solid consists of array of atoms in close proximity. This array may be periodic, as in a crystal lattice, but this is not essential. Each atom consists of a positive nucleus surrounded by neutralizing set of electrons and preforms a many-body system. The Hamiltonian of a solid can be written as follows [35]:

$$
\begin{aligned}
H= & \sum_{i} \frac{-\hbar^{2} \nabla_{i}^{2}}{2 M_{i}}+\frac{1}{2} \sum_{i, j}^{\prime} \frac{Z^{2} e^{2}}{\left|\vec{R}_{i}-\vec{R}_{j}\right|}+\sum_{k} \frac{-\hbar^{2} \nabla_{k}^{2}}{2 m} \\
& +\frac{1}{2} \sum_{k, l}^{\prime} \frac{e^{2}}{\left|\vec{r}_{k}-\vec{r}_{l}\right|}-\sum_{k, i} \frac{Z e^{2}}{\left|\vec{r}_{k}-\vec{R}_{i}\right|},
\end{aligned}
$$

where the nuclear positions are given by $\left(\vec{R}_{i}, \vec{R}_{j}\right)$ and the electron positions are given by $\left(\vec{r}_{k}, \vec{r}_{l}\right) ; Z$ is nuclear charge and $(M, m)$ are the masses of the nucleus and electron, respectively. The primes on the summations mean that we must exclude the terms $i=j, \ldots$ to prevent self-interactions. Within the Born-Oppenheimer approximation, we have (see [35], for details)

$$
H=\sum_{k} \frac{-\hbar^{2}}{2 m} \nabla_{k}^{2}+\frac{1}{2} \sum_{k, l}^{\prime} \frac{e^{2}}{\left|\vec{r}_{k}-\vec{r}_{l}\right|}-\sum_{k, i} \frac{Z e^{2}}{\left|\vec{r}_{k}-\vec{R}_{i}\right|} .
$$

The second term of this relation shows the interaction between electrons that is the only obstacle to use singleparticle wave function. Within Hartree's approximation we replace the interaction term

$$
V_{\text {INT }}\left(\vec{r}_{1}, \ldots, \vec{r}_{N}\right)=\frac{1}{2} \sum_{i, j}^{\prime} \frac{e^{2}}{\left|\vec{r}_{i}-\vec{r}_{j}\right|}
$$

by an approximate form

$$
\bar{V}_{\mathrm{INT}}\left(\vec{r}_{1}, \ldots, \vec{r}_{N}\right)=\sum_{i} V_{i}\left(\vec{r}_{i}\right)
$$

Now we focus on correlation effects and the Thomas-Fermi model in a solid in equilibrium. Consider a system that includes electronic gas in equilibrium. In this system the local electron density is determined by the Fermi level which is a property of the solid as a whole. Suppose we apply a local electrostatic force that disturbs the system. In this situation, there is a tendency for electrons to migrate until the density is again consistent with the Fermi level. Suppose that $\mathscr{U}_{0}(r)$ is 
the potential of the applied electrostatic force and $V(r)$ is the electrostatic potential due to movement of charges. Then the total potential of the system $\mathcal{U}(r)$ can be obtained from the following relation:

$$
\mathcal{U}(r)=\mathscr{U}_{0}(r)+V(r) .
$$

The electrostatic potential satisfies Poisson's equation with the change in the charge density $\rho(r)$ :

$$
\nabla^{2} V(r)=4 \pi \rho(r)
$$

where $\rho(r)$ is given by

$$
\rho(r)=e \mathcal{U}(r) N\left(E_{f}\right)
$$

and $N\left(E_{f}\right)$ is the density of states at the Fermi energy. Thus we have

$$
\nabla^{2}\left(\mathcal{U}(r)-\mathcal{U}_{0}(r)\right)=\lambda^{2} \mathcal{U}(r)
$$

where

$$
\lambda^{2} \equiv 4 \pi e N\left(E_{f}\right)
$$

Now (22) can be rewritten as

$$
\left(\nabla^{2}-\lambda^{2}\right) \mathcal{U}(r)=\nabla^{2} \mathcal{U}_{0}(r)
$$

The corresponding homogeneous Thomas-Fermi equation is

$$
\left(\nabla^{2}-\lambda^{2}\right) \mathcal{U}(r)=0 .
$$

By using this equation we calculate the total potential $\mathcal{U}(r)$ and then we are able to obtain a relation for interaction potential. In what follows we are going to investigate ThomasFermi model firstly in the presence of a minimal measurable length encoded in a generalized uncertainty principle. We obtain total potential, interaction potential, and the correlation energy that is the rate of required energy for putting electron in the correlation hole and at the end we will find a relation for generalized dielectric function. In each step we compare our results with the standard case to see the effects of quantum gravity corrections. We note that the GUP as a manifestation of quantum gravity effects reflects the universality of quantum gravity corrections [36]. So, all quantum mechanical systems are affected by the quantum gravity effects and Thomas-Fermi model for many-body quantum interactions is not an exception in this regard. On the other hand, quantum gravity effects may have considerable effects on many-body quantum systems and one cannot neglect these effects in principle. It is important to note also that incorporation of the GUP effects in many-body problems may open new window to test quantum gravity in the lab.

\section{Thomas-Fermi Equation in the Presence of Minimal Length}

In one-dimensional case the deformed Heisenberg algebra reads

$$
[X, P]=i \hbar\left(1+\beta P^{2}\right)
$$

One of the possible representations of this algebra can be written as

$$
P=p\left(1+\frac{\beta}{3} p^{2}\right), \quad X=x,
$$

where $x$ and $p$ are the conventional position and momentum operators. Note that while the existence of the natural cutoffs such as the minimal length is a common feature of all quantum gravity candidates, the form of the GUP containing this minimal length is model-dependent. In principle, these representations are not unique. For instance, we have supposed $\langle p\rangle=0$ in these representations and we have taken into account only terms of the first order in $\beta$ in a perturbative expansion. Since $p=-i \hbar / \vec{\nabla}$, so the deformed gradient operator $\left(\overrightarrow{\nabla^{\prime}}\right)$ will be as follows:

$$
\overrightarrow{\nabla^{\prime}}=\vec{\nabla}\left(1-\frac{\beta}{3} \hbar^{2} \nabla^{2}\right) .
$$

Up to the first order in $\beta$ we have

$$
\nabla^{\prime 2}=\overrightarrow{\nabla^{\prime}} \cdot \overrightarrow{\nabla^{\prime}}=\nabla^{2}-\frac{2 \beta \hbar^{2}}{3} \nabla^{4} .
$$

Now in deformed space (25) is replaced with

$$
\left(\nabla^{\prime 2}-\lambda^{2}\right) \mathcal{U}^{\prime}(r)=0
$$

where $\mathcal{U}^{\prime}(r)$ is the total potential in deformed space. Therefore we find the following differential equation to first order in $\beta$ :

$$
\left(-\frac{2 \beta \hbar^{2}}{3} \nabla^{4}+\nabla^{2}-\lambda^{2}\right) \mathcal{U}^{\prime}(r)=0 .
$$

We suppose the system is spherically symmetric so that there is no angular dependence and therefore

$$
\begin{aligned}
& \nabla^{2}=\frac{1}{r^{2}} \frac{\partial}{\partial r}\left(r^{2} \frac{\partial}{\partial r}\right)=\frac{\partial^{2}}{\partial r^{2}}+\frac{2}{r} \frac{\partial}{\partial r}, \\
& \nabla^{4}=\nabla^{2} \cdot \nabla^{2}=\frac{\partial^{4}}{\partial r^{4}}+\frac{4}{r} \frac{\partial^{3}}{\partial r^{3}} .
\end{aligned}
$$

Now (31) takes the following form:

$$
\begin{aligned}
& \frac{d^{4} \mathcal{U}^{\prime}(r)}{d r^{4}}+\frac{4}{r} \frac{d^{3} \mathcal{U}^{\prime}(r)}{d r^{3}}-\left(\frac{3}{2 \beta \hbar^{2}}\right) \frac{d^{2} \mathcal{U}^{\prime}(r)}{d r^{2}} \\
& -\left(\frac{3}{\beta \hbar^{2} r}\right) \frac{d \mathcal{U}^{\prime}(r)}{d r}+\left(\frac{3 \lambda^{2}}{2 \beta \hbar^{2}}\right) \mathcal{U}^{\prime}(r)=0 .
\end{aligned}
$$

This differential equation has the following solutions:

$$
\begin{aligned}
\mathcal{U}^{\prime}(r)= & C_{1} e^{(1 / 2)\left(-r \sqrt{\left(3-\sqrt{9-24 \hbar^{2} \beta \lambda^{2}}\right) / \hbar^{2} \beta}-2 \ln (r)\right)} \\
& +C_{2} e^{(1 / 2)\left(r \sqrt{\left(3-\sqrt{9-24 \hbar^{2} \beta \lambda^{2}}\right) / \hbar^{2} \beta}-2 \ln (r)\right)} \\
& +C_{3} e^{(1 / 2)\left(-r \sqrt{\left(3+\sqrt{9-24 \hbar^{2} \beta \lambda^{2}}\right) / \hbar^{2} \beta}-2 \ln (r)\right)} \\
& +C_{4} e^{(1 / 2)\left(r \sqrt{\left(3+\sqrt{9-24 \hbar^{2} \beta \lambda^{2}}\right) / \hbar^{2} \beta}-2 \ln (r)\right)} .
\end{aligned}
$$


For a given $\beta$ with $\beta<9 / 24 \hbar^{2} \lambda^{2}$, the terms with coefficients $C_{2}$ and $C_{4}$ are not bounded in the limit of $r \rightarrow \infty$. So, to have correct limiting case we set $C_{2}=C_{4}=0$. On the other hand, only the first term gives the physically appropriate limiting result (note also that the term containing $C_{3}$ has no suitable limit; that is, in the limit of $\beta \rightarrow 0$, it cannot recover the standard result in the absence of GUP). So we consider just this term and we set $C_{1}=e$, where $e$ is the electron charge. We define

$$
\lambda^{\prime} \equiv \frac{1}{2} \sqrt{\frac{3-\sqrt{9-24 \hbar^{2} \beta \lambda^{2}}}{\hbar^{2} \beta}} .
$$

So, we find

$$
\mathcal{U}^{\prime}(r)=e \frac{e^{-\lambda^{\prime} r}}{r}
$$

This relation gives the effective interaction potential between electrons in Thomas-Fermi model in the presence of minimal measurable length. It is obvious that, in the limit of $\beta \rightarrow 0, \lambda^{\prime}$ should be transformed into the $\lambda$. In fact, the limit of $\beta \rightarrow 0$ can be calculated easily. The square root

$$
\frac{1}{2} \sqrt{\frac{3-\sqrt{9-24 \hbar^{2} \beta \lambda^{2}}}{\hbar^{2} \beta}}
$$

can be expanded as

$$
\begin{aligned}
\frac{1}{2} \sqrt{\frac{3-3 \sqrt{1-(8 / 3) \hbar^{2} \beta \lambda^{2}}}{\hbar^{2} \beta}} & =\frac{1}{2} \sqrt{\frac{3-3\left(1-(8 / 3) \hbar^{2} \beta \lambda^{2}\right)^{1 / 2}}{\hbar^{2} \beta}} \\
& \approx \frac{1}{2} \sqrt{\frac{3-3\left(1-(8 / 6) \hbar^{2} \beta \lambda^{2}\right)}{\hbar^{2} \beta}} .
\end{aligned}
$$

The limit of this expression for $\beta \rightarrow 0$ gives $\lambda$ as required. It could then be introduced, instead of the normal interaction potential equation (17), into the Schrödinger equation

$$
V_{\mathrm{INT}}\left(\vec{r}_{1}, \ldots, \vec{r}_{N}\right) \longrightarrow \frac{1}{2} \sum_{i, j}^{\prime} e^{2} \frac{e^{-\lambda^{\prime}\left|\vec{r}_{i}-\vec{r}_{j}\right|}}{\left|\vec{r}_{i}-\vec{r}_{j}\right|}
$$

This induced potential causes the correlation energy which an electron needs in order to be located at the correlation hole. In solid state physics, to find this energy we use the following relation [35]:

$$
E_{\mathrm{CORR}}=\lim _{r \rightarrow 0}\left(-\frac{e}{2} V_{\mathrm{IND}}(r)\right)
$$

where

$$
V_{\mathrm{IND}}(r) \equiv \mathcal{U}^{\prime}(r)-\mathcal{U}^{\prime}(r \longrightarrow 0) .
$$

Using (36) we have

$$
V_{\mathrm{IND}}(r)=-\frac{e}{2}\left(1-e^{-\lambda^{\prime} r}\right)
$$

Therefore, we find

$$
E_{\mathrm{CORR}}=-\lambda^{\prime} \frac{e^{2}}{2}
$$

Note that the effect of GUP is hidden in the definition of $\lambda^{\prime}$. This energy appears as an additive constant to the energy of each electron.

\section{The Generalized Dielectric Function in Deformed Space}

In Thomas-Fermi model, the total potential $\mathscr{U}(r)$ is obtained from a known external potential $\mathscr{U}_{0}(r)$. In classical electrostatics, we can introduce a dielectric constant that expresses the relationship between these two potentials:

$$
\mathscr{U} \approx \frac{\mathscr{U}_{0}}{\epsilon} .
$$

It would be correct also to describe this potential in terms of a dielectric function $\epsilon(r)$ :

$$
\mathcal{U}(r)=\frac{\mathscr{U}_{0}(r)}{\epsilon(r)} .
$$

Now we come back to (24) and write it in deformed space as

$$
\left(\nabla^{\prime 2}-\lambda^{2}\right) \mathcal{U}(r)=\nabla^{\prime 2} \mathscr{U}_{0}(r),
$$

where

$$
\nabla^{\prime 2}=\nabla^{2}-\frac{2 \beta \hbar^{2}}{3} \nabla^{4}
$$

So we find

$$
\begin{gathered}
\nabla^{2} \mathcal{U}(r)-\frac{2 \beta \hbar^{2}}{3} \nabla^{4} \mathcal{U}(r)-\lambda^{2} \mathcal{U}(r) \\
=\nabla^{2} \mathcal{U}_{0}(r)-\frac{2 \beta \hbar^{2}}{3} \nabla^{4} \mathcal{U}_{0}(r) .
\end{gathered}
$$

Taking Fourier transform and using a wave vector-dependent dielectric function $\epsilon(\vec{q})$ we can write

$$
\begin{gathered}
\mathcal{U}(\vec{r})=\sum_{\vec{q}} \mathcal{U}(\vec{q}) e^{i \vec{q} \cdot \vec{r}}, \\
\mathcal{U}_{0}(\vec{r})=\sum_{\vec{q}} \mathcal{U}_{0}(\vec{q}) e^{i \overrightarrow{q_{0}} \cdot \vec{r}} .
\end{gathered}
$$

By substituting these relations in (48) we find

$$
\left[-q^{2}-\frac{2 \beta \hbar^{2} q^{4}}{3}-\lambda^{2}\right] \mathcal{U}(\vec{q})=\left[-q^{2}-\frac{2 \beta \hbar^{2} q^{4}}{3}\right] \mathcal{U}_{0}(\vec{q}) .
$$

So, we find

$$
\left[1+\frac{\lambda^{2}}{q^{2}+\left(2 \beta \hbar^{2} q^{4} / 3\right)}\right] \mathcal{U}(\vec{q})=\mathscr{U}_{0}(\vec{q}) .
$$


Therefore,

$$
\mathcal{U}(\vec{q})=\frac{\mathscr{U}_{0}(\vec{q})}{1+\left(\lambda^{2} /\left(q^{2}+\left(2 \beta \hbar^{2} q^{4} / 3\right)\right)\right)} .
$$

From relation $\mathcal{U}(\vec{q})=\mathcal{U}_{0}(\vec{q}) / \epsilon(\vec{q})$, the generalized dielectric function in deformed space is obtained as

$$
\epsilon(\vec{q})=1+\frac{\lambda^{2}}{q^{2}\left(1+(2 \beta / 3) \hbar^{2} q^{2}\right)} .
$$

To first order in $\beta$ and with $\hbar=1$, we can conclude that $\vec{q}$ in deformed space is defined as

$$
\vec{q} \rightarrow \overrightarrow{q^{\prime}}=\vec{q}\left(1+\frac{\beta}{3} q^{2}\right)
$$

Therefore,

$$
\epsilon(\vec{q})=1+\frac{\lambda^{2}}{q^{2}\left(1+(2 \beta / 3) q^{2}\right)} .
$$

It is important to note that corrections to the dielectric function in deformed space are wave number dependent. By the Fourier transform of $\mathcal{U}(\vec{q})$, we can calculate the total potential $\mathscr{U}(r)$ to find

$$
\mathcal{U}(r)=\frac{1}{(2 \pi)^{3}} \int \frac{d^{3} q}{1+\beta q^{2}}\left(\frac{\mathcal{U}_{0}(\vec{q})}{\epsilon(\vec{q})}\right) e^{i \vec{q} \cdot \vec{r}} .
$$

Since

$$
\mathscr{U}_{0}(r)=\frac{e}{|\vec{r}|}
$$

the Fourier transform of this potential is

$$
\mathscr{U}_{0}\left(\overrightarrow{q^{\prime}}\right)=\int d^{3} r \mathscr{U}_{0}(r) e^{-i q^{\prime} \cdot \vec{r}}=\int 4 \pi r^{2} d r \frac{e}{r} e^{-i q^{\prime} \cdot r} ;
$$

thus we find

$$
\mathscr{U}_{0}\left(\overrightarrow{q^{\prime}}\right)=\frac{-4 \pi e}{q^{\prime 2}}
$$

By substituting $\overrightarrow{q^{\prime}}=\vec{q}\left(1+(\beta / 3) q^{2}\right)$ in this relation we find

$$
\mathscr{U}_{0}(\vec{q})=\frac{-4 \pi e}{q^{2}\left(1+(\beta / 3) q^{2}\right)^{2}} .
$$

To first order in $\beta$ this equation can be written as

$$
\mathcal{U}_{0}(\vec{q})=\frac{-4 \pi e}{q^{2}\left(1+(2 \beta / 3) q^{2}\right)} .
$$

From (55) and (61) we can obtain the total potential as follows:

$$
\begin{array}{r}
\mathcal{U}(\vec{r})=\frac{-4 \pi e}{(2 \pi)^{3}} \int \frac{d^{3} q}{1+\beta q^{2}}\left(\frac{1}{q^{2}\left(1+(2 \beta / 3) q^{2}\right)+\lambda^{2}}\right) e^{i \vec{q} \cdot \vec{r}}, \\
d^{3} q=4 \pi q^{2} d q ;
\end{array}
$$

thus,

$$
\begin{aligned}
\mathcal{U}(\vec{r})= & \frac{-(4 \pi)^{2} e}{(2 \pi)^{3}} \\
& \times \int \frac{d q}{1+\beta q^{2}}\left(\frac{q^{2}}{q^{2}\left(1+(2 \beta / 3) q^{2}\right)+\lambda^{2}}\right) e^{i \vec{q} \cdot \vec{r}} .
\end{aligned}
$$

Figure 1 shows the real part and also the norm of $\mathcal{U}(r)$ for different values of $\beta$. We note that, since GUP comes from quantum gravity effects via a perturbational scheme, it is natural to think that the GUP parameter is a very small parameter. The case with $\beta=0$ is the standard case and any deviation from this value incorporates the quantum gravitational effects. We have chosen the mentioned values of $\beta$ in figure just as examples and there is no reason to prevent selection of other nonvanishing values for $\beta$.

The correlation energy can be expressed in the form of

$$
E_{\mathrm{CORR}}=\lim _{r \rightarrow 0}\left(-\frac{e}{2} V_{\mathrm{IND}}(\vec{r})\right)
$$

where

$$
V_{\mathrm{IND}}(\vec{r})=\frac{1}{(2 \pi)^{3}} \int \frac{d^{3} q}{1+\beta q^{2}} V_{\mathrm{IND}}(\vec{q}) e^{i \vec{q} \cdot \vec{r}}
$$

The Fourier transform of the $V_{\mathrm{IND}}(\vec{r})$ is as follows:

$$
\begin{aligned}
V_{\mathrm{IND}}(\vec{q}) & =\mathcal{U}(\vec{q})-\mathcal{U}(\vec{q} \longrightarrow 0) \\
& =\mathcal{U}_{0}(\vec{q})\left(\frac{1}{\epsilon(\vec{q})}-1\right) .
\end{aligned}
$$

Thus,

$$
\begin{aligned}
E_{\mathrm{CORR}}= & \lim _{r \rightarrow 0} \frac{-e}{2(2 \pi)^{3}} \\
& \times \int \frac{d^{3} q}{1+\beta q^{2}}\left(\frac{-4 \pi e}{q^{2}\left(1+(2 \beta / 3) q^{2}\right)}\right.
\end{aligned}
$$

$$
\times((1 / \epsilon(q))-1)) e^{i \vec{q} \cdot \vec{r}}
$$

$$
\begin{aligned}
E_{\mathrm{CORR}}= & \lim _{r \rightarrow 0} \frac{(4 \pi e)^{2}}{2(2 \pi)^{3}} \\
& \times \int \frac{q^{2} d q}{1+\beta q^{2}}\left(\frac{1}{q^{2}\left(1+(2 \beta / 3) q^{2}\right)+\lambda^{2}}\right.
\end{aligned}
$$

$$
\left.-\frac{1}{q^{2}\left(1+(2 \beta / 3) q^{2}\right)}\right) e^{i \vec{q} \cdot \vec{r}}
$$

This integral can be calculated numerically to obtain $E_{\mathrm{CORR}}$. Figure 2 shows the real part and also the norm of $E_{\mathrm{CORR}}$ for different values of $\beta$. 


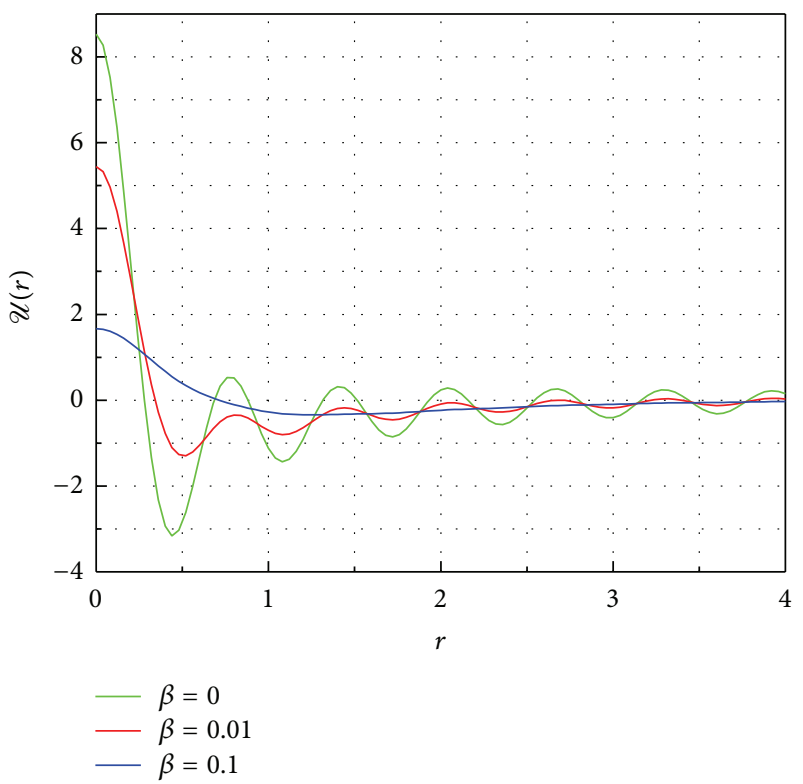

(a)

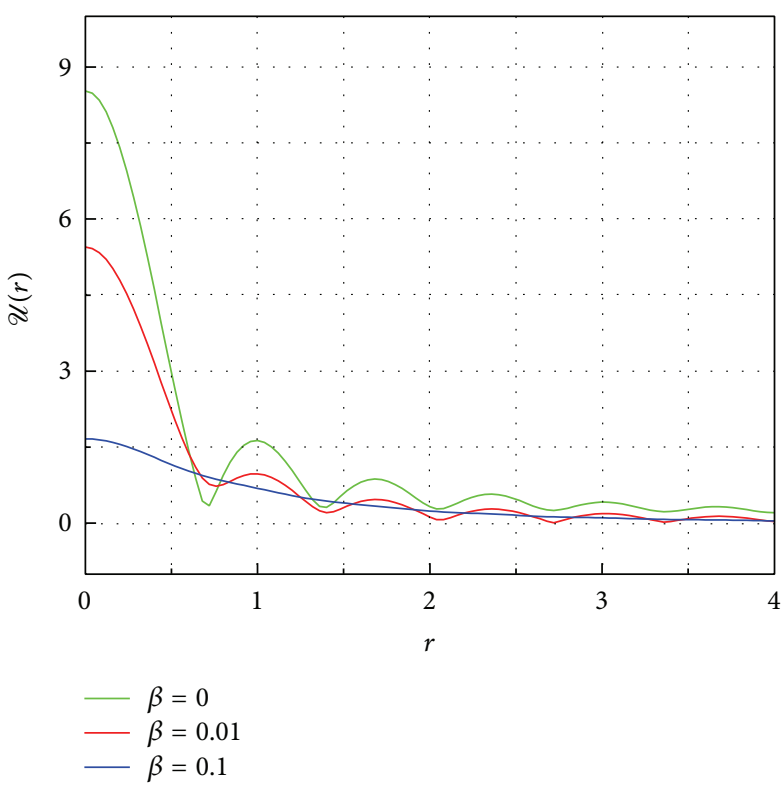

(b)

FIGURE 1: Real part (a) and norm (b) of $\mathcal{U}(r)$ versus $r$ for different values of $\beta$.

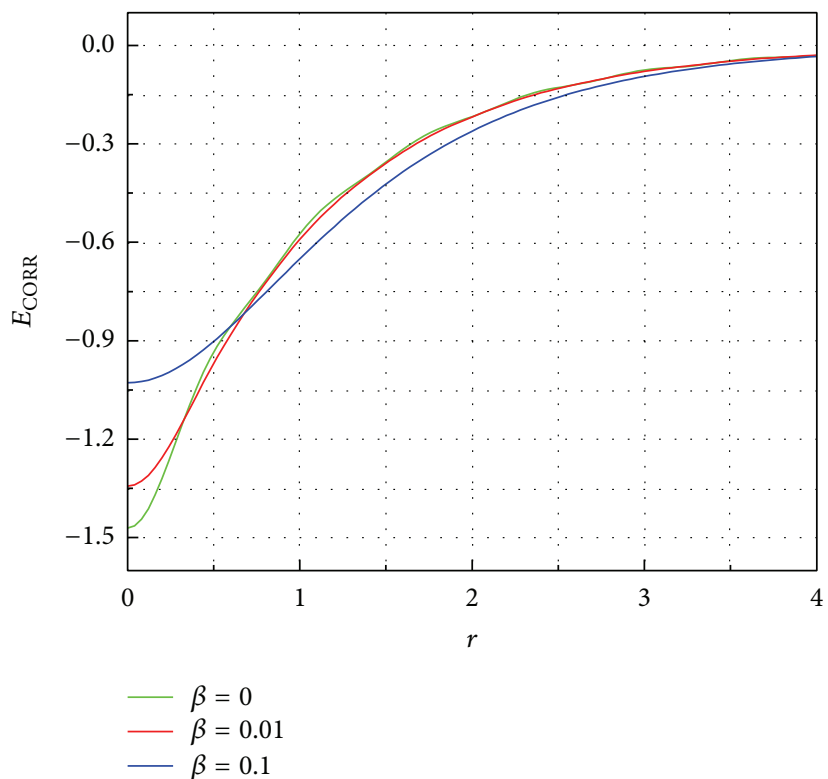

(a)

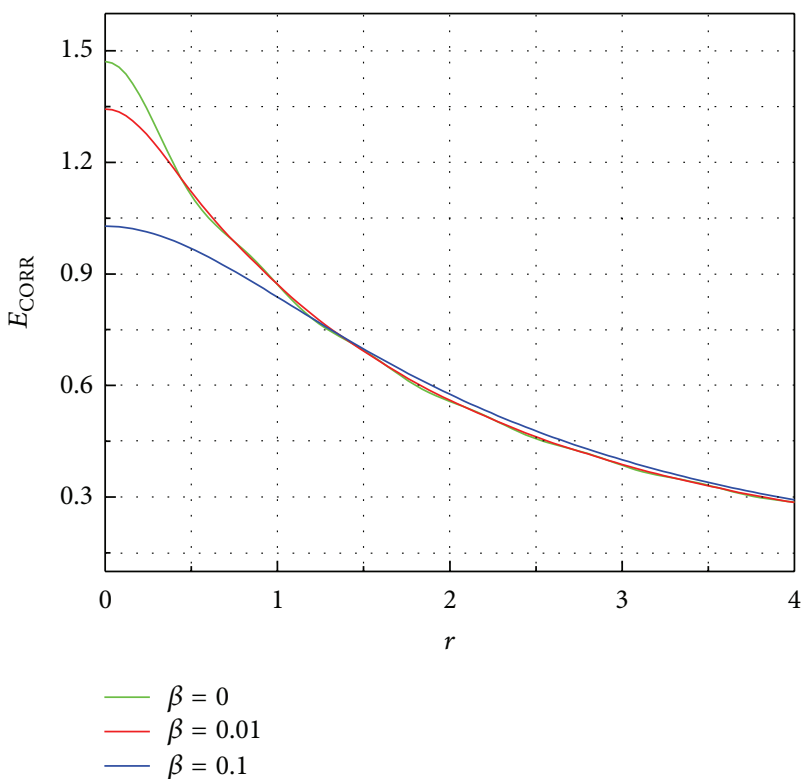

(b)

FIGURE 2: Real part (a) and norm (b) of $E_{\text {CORR }}$ for different values of $\beta$.

\section{Thomas-Fermi Model in the Presence of Minimal Length and Maximal Momentum}

Magueijo and Smolin have shown that in the context of the doubly special relativity a test particle's momentum cannot be arbitrarily imprecise and therefore there is an upper bound for momentum fluctuation [28-30] (see also [31]). Then it has been shown that this may lead nontrivially to a maximal measurable momentum for a test particle $[32,33]$. In this framework, the GUP that predicts both a minimal observable length and a maximal momentum for a test particle can be written as follows [20,32-34]:

$$
\Delta X \Delta P \geq \frac{\hbar}{2}\left(1-2 \beta\langle P\rangle+4 \beta^{2}\left\langle P^{2}\right\rangle\right)
$$

or since $\Delta P \equiv P-\langle P\rangle$,

$$
\Delta X \Delta P \geq \frac{\hbar}{2}\left[1-\beta(\Delta P)+2 \beta^{2}(\Delta P)^{2}\right] .
$$


In this framework the following algebraic structure can be deduced (see $[32,33]$ ):

$$
[X, P]=i \hbar\left(1-\beta P+2 \beta^{2} P^{2}\right),
$$

where $\beta$ is the GUP parameter in the presence of both a minimal length and a maximal momentum. Similar to the minimal length case, we can define $[20,34]$

$$
\begin{gathered}
X=x \\
P=p\left(1-\beta p+\frac{\beta^{2}}{3} p^{2}\right),
\end{gathered}
$$

where, as before, $x$ and $p$ satisfy the canonical commutation relations via the Jacobi identity and $X$ and $P$ satisfy the generalized commutation relations in the presence of minimal length and maximal momentum [20,32, 33]:

$$
[X, P]=i \hbar\left(1-\beta p+\frac{\beta^{2}}{3} p^{2}\right)
$$

In this section we want to calculate the effects of both minimal length and maximal momentum on the total potential and generalized dielectric function in Thomas-Fermi model. In this case the deformed momentum is defined as

$$
P \longrightarrow P^{\prime}=p\left(1-\beta p+\frac{\beta^{2}}{3} p^{2}\right) \text {. }
$$

So the gradient operator will change as follows:

$$
\begin{gathered}
-i \hbar \vec{\nabla} \longrightarrow-i \hbar \vec{\nabla}\left[1-\beta(-i \hbar \nabla)+\frac{\beta^{2}}{3}(-i \hbar \nabla)^{2}\right] . \\
\vec{\nabla} \longrightarrow \vec{\nabla}^{\prime}=\vec{\nabla}\left(1+i \hbar \beta \nabla-\frac{\beta^{2}}{3} \hbar^{2} \nabla^{2}\right)
\end{gathered}
$$

To first order in $\beta, \nabla^{\prime 2}$ can be written as

$$
\nabla^{\prime 2}=\vec{\nabla}^{\prime} \cdot \vec{\nabla}^{\prime}=\nabla^{2}+2 i \beta^{3} \hbar \nabla^{3}-\frac{2 \beta^{4} \hbar^{2}}{3} \nabla^{4}
$$

Now, the Thomas-Fermi equation $\left(\nabla^{2}-\lambda^{2}\right) \mathcal{U}(r)=0$ in this case transforms to the following equation:

$$
\left(-\frac{2 \beta^{4} \hbar^{2}}{3} \nabla^{4}+2 i \beta^{3} \hbar \nabla^{3}+\nabla^{2}-\lambda^{2}\right) \mathcal{U}(r)=0 .
$$

To solve this equation we assume $\lambda$ to be a complex quantity so that

$$
\lambda=\lambda_{1}+i \lambda_{2}, \quad \lambda^{2}=\lambda_{1}^{2}-\lambda_{2}^{2}+2 i \lambda^{*}
$$

where $\lambda^{*} \equiv \lambda_{1} \lambda_{2}$. Then the total potential will be a complex function as

$$
\mathcal{U}(r)=\mathcal{U}_{R}+i \mathcal{U}_{I} \text {. }
$$

In spherical coordinate

$$
\nabla^{3}=\frac{\partial^{3}}{\partial r^{3}}+\frac{4}{r} \frac{\partial^{2}}{\partial r^{2}}+\frac{2}{r^{2}} \frac{\partial}{\partial r}
$$

and using (32), we find

$$
\left[-\frac{2 \beta^{4} \hbar^{2}}{3} \nabla^{4}+\nabla^{2}-\left(\lambda_{1}^{2}-\lambda_{2}^{2}\right)+i\left(-2 \lambda^{*}+2 \beta^{3} \hbar \nabla^{3}\right)\right] \mathcal{U}(r)=0 ;
$$

therefore,

$$
\begin{aligned}
& {\left[\frac{d^{4} \mathcal{U}(r)}{d r^{4}}+\frac{4}{r} \frac{d^{3} \mathscr{U}(r)}{d r^{3}}-\frac{3}{2 \beta \hbar^{2}} \frac{d^{2} \mathscr{U}(r)}{d r^{2}}\right.} \\
& \left.-\frac{3}{\beta \hbar^{2} r} \frac{d \mathcal{U}(r)}{d r}+\frac{3}{2 \beta \hbar^{2}}\left(\lambda_{1}^{2}-\lambda_{2}^{2}\right) \mathcal{U}(r)\right] \\
& +i\left[-\frac{3}{\hbar} \frac{d^{3} \mathcal{U}(r)}{d r^{3}}-\frac{12}{\hbar r} \frac{d^{2} \mathcal{U}(r)}{d r^{2}}\right. \\
& \left.-\frac{6}{\hbar r^{2}} \frac{d \mathcal{U}(r)}{d r}+\frac{3 \lambda^{*}}{\beta \hbar^{2}} \mathcal{U}(r)\right]=0 .
\end{aligned}
$$

The real part of this complex differential equation can be solved to obtain

$$
\begin{aligned}
\mathcal{U}_{R}(r)= & C_{1} e^{(1 / 2)\left(-r \sqrt{\left(\left(3-\sqrt{9-24 \hbar^{2} \beta \lambda^{\prime}}\right) / \hbar^{2} \beta\right)}-2 \ln (r)\right)} \\
& +C_{2} e^{(1 / 2)\left(r \sqrt{\left(\left(3-\sqrt{9-24 \hbar^{2} \beta \lambda^{\prime}}\right) / \hbar^{2} \beta\right)}-2 \ln (r)\right)} \\
& +C_{3} e^{(1 / 2)\left(-r \sqrt{\left(\left(3+\sqrt{9-24 \hbar^{2} \beta \lambda^{\prime}}\right) / \hbar^{2} \beta\right)}-2 \ln (r)\right)} \\
& +C_{4} e^{(1 / 2)\left(r \sqrt{\left(\left(3+\sqrt{9-24 \hbar^{2} \beta \lambda^{\prime}}\right) / \hbar^{2} \beta\right)}-2 \ln (r)\right)},
\end{aligned}
$$

where by definition

$$
\lambda^{\prime} \equiv \lambda_{1}^{2}-\lambda_{2}^{2}
$$

Only the first term of the solution (82) satisfies the required boundary conditions and gives correct limiting results

$$
\mathcal{U}_{R}(r)=C_{1} e^{-(r / 2) \sqrt{\left(3-\sqrt{9-24 \hbar^{2} \beta\left(\lambda_{1}^{2}-\lambda_{2}^{2}\right)}\right) / \hbar^{2} \beta}} e^{-\ln (r)} .
$$

We suppose $C_{1}=e$ to find

$$
\mathcal{U}_{R}(r)=\frac{e^{-(r / 2) \sqrt{\left(3-\sqrt{9-24 \hbar^{2} \beta\left(\lambda_{1}^{2}-\lambda_{2}^{2}\right)}\right) / \hbar^{2} \beta}}}{r} .
$$

We note that the limit of $\beta \rightarrow 0$ can be obtained easily much similar to the analysis that has been done just after (36).

In this case the effective interaction potential between electrons in the Thomas-Fermi model takes the following form:

$$
\begin{aligned}
& V_{\mathrm{INT}}\left(\vec{r}_{1}, \ldots, \vec{r}_{N}\right) \\
& \longrightarrow \frac{1}{2} \sum_{i, j}^{\prime} e^{2} \frac{e^{-\left((1 / 2) \sqrt{\left(3-\sqrt{9-24 \hbar^{2} \beta\left(\lambda_{1}^{2}-\lambda_{2}^{2}\right)}\right) / \hbar^{2} \beta}\right)\left|r_{i}-r_{j}\right|}}{\left|r_{i}-r_{j}\right|},
\end{aligned}
$$


and the correlation energy is

$$
E_{\mathrm{CORR}}=\lim _{r \rightarrow 0}\left(-\frac{e}{2} V_{\mathrm{IND}}(r)\right)
$$

so that

$$
\begin{gathered}
V_{\mathrm{IND}}(r)=-\frac{e}{r}\left(1-e^{-(r / 2) \sqrt{\left(3-\sqrt{9-24 \hbar^{2} \beta\left(\lambda_{1}^{2}-\lambda_{2}^{2}\right)}\right) / \hbar^{2} \beta}}\right), \\
E_{\mathrm{CORR}}=-\frac{e^{2}}{2}\left(\frac{1}{2} \sqrt{\left(3-\sqrt{9-24 \hbar^{2} \beta\left(\lambda_{1}^{2}-\lambda_{2}^{2}\right)}\right) / \hbar^{2} \beta}\right) .
\end{gathered}
$$

To calculate the generalized dielectric function in the presence of both a minimal length and a maximal momentum, we proceed in the same manner as in the previous section. Starting with

$$
\left(\nabla^{2}-\lambda^{2}\right) \mathcal{U}(r)=\nabla^{2} \mathcal{U}_{0}(r)
$$

now the gradient operator changes as follows:

$$
\nabla^{2} \rightarrow \nabla^{\prime 2}=\nabla^{2}+2 i \beta^{3} \hbar \nabla^{3}-\frac{2 \beta^{4} \hbar^{2}}{3} \nabla^{4}
$$

so we find

$$
\begin{gathered}
\left(\nabla^{2}+2 i \beta^{3} \hbar \nabla^{3}-\frac{2 \beta^{4} \hbar^{2}}{3} \nabla^{4}-\lambda^{2}\right) \mathcal{U}(r) \\
=\left(\nabla^{2}+2 i \beta^{3} \hbar \nabla^{3}-\frac{2 \beta^{4} \hbar^{2}}{3} \nabla^{4}\right) \mathcal{U}_{0}(r),
\end{gathered}
$$

where

$$
\lambda^{2}=\lambda_{1}^{2}-\lambda_{2}^{2}+2 i \lambda^{*}
$$

Thus, we have

$$
\begin{aligned}
\nabla^{2} \mathcal{U}(r) & -\frac{2 \beta^{4} \hbar^{2}}{3} \nabla^{4} \mathcal{U}(r)-\left(\lambda_{1}^{2}-\lambda_{2}^{2}\right) \mathcal{U}(r) \\
& +i\left(2 \beta^{3} \hbar \nabla^{3} \mathcal{U}(r)-2 \lambda^{*} \mathcal{U}(r)\right) \\
= & \nabla^{2} \mathcal{U}_{0}(r)-\frac{2 \beta^{4} \hbar^{2}}{3} \nabla^{4} \mathcal{U}_{0}(r)+i\left(2 \beta^{3} \hbar \nabla^{3} \mathcal{U}_{0}(r)\right) .
\end{aligned}
$$

Taking the Fourier transforms of $\mathcal{U}(r)$ and $\mathcal{U}_{0}(r)$, we find

$$
\begin{aligned}
& \left(-q^{2}\right) \mathcal{U}(\vec{q})-\frac{2 \beta^{4} \hbar^{2}}{3}\left(q^{4}\right) \mathcal{U}(\vec{q})-\left(\lambda_{1}^{2}-\lambda_{2}^{2}\right) \mathcal{U}(\vec{q}) \\
& \quad+i\left[2 \beta^{3} \hbar\left(-i q^{3}\right) \mathcal{U}(\vec{q})-2 \lambda^{*} \mathcal{U}(\vec{q})\right] \\
& =\left(-q^{2}\right) \mathcal{U}_{0}(\vec{q})-\frac{2 \beta^{4} \hbar^{2}}{3}\left(q^{4}\right) \mathcal{U}_{0}(\vec{q}) \\
& \quad+i\left[2 \beta^{3} \hbar\left(-i q^{3}\right) \mathcal{U}_{0}(\vec{q})\right]
\end{aligned}
$$

which gives

$$
\left[-q^{2}-\frac{2 \beta^{4}}{3} \hbar^{2} q^{4}-\left(\lambda_{1}^{2}-\lambda_{2}^{2}\right)+2 \beta^{3} \hbar q^{3}-2 i \lambda^{*}\right] \mathcal{U}(\vec{q})
$$

$$
=\left[-q^{2}-\frac{2 \beta^{4}}{3} \hbar^{2} q^{4}+2 \beta^{3} \hbar q^{3}\right] \mathcal{U}_{0}(\vec{q}) \text {. }
$$

So we find

$$
\begin{gathered}
{\left[-q^{2}-\frac{2 \beta^{4}}{3} \hbar^{2} q^{4}+2 \beta^{3} \hbar q^{3}-\lambda^{2}\right] \mathcal{U}(\vec{q})} \\
=\left[-q^{2}-\frac{2 \beta^{4}}{3} \hbar^{2} q^{4}+2 \beta^{3} \hbar q^{3}\right] \mathcal{U}_{0}(\vec{q}) .
\end{gathered}
$$

This gives finally

$$
\left[1+\frac{\lambda^{2}}{q^{2}-2 \hbar \beta^{3} q^{3}+\left(2 \beta^{4} \hbar^{2} q^{4} / 3\right)}\right] \mathcal{U}(\vec{q})=\mathscr{U}_{0}(\vec{q})
$$

or

$$
\mathcal{U}(\vec{q})=\frac{\mathscr{U}_{0}(\vec{q})}{1+\left(\lambda^{2} /\left(q^{2}-2 \hbar \beta^{3} q^{3}+\left(2 \beta^{4} \hbar^{2} q^{4} / 3\right)\right)\right)} .
$$

From the relation $\mathscr{U}(\vec{q})=\mathscr{U}_{0}(\vec{q}) / \epsilon(\vec{q})$, we can conclude that the generalized dielectric function in the presence of a minimal length and a maximal momentum is as follows:

$$
\epsilon(q)=1+\frac{\lambda^{2}}{q^{2}\left(1+(2 \beta / 3) \hbar^{2} q^{2}-2 \beta \hbar q\right)} .
$$

By setting $\hbar=1$ and considering only the terms that are first order in $\beta$, we find

$$
\vec{q} \rightarrow \overrightarrow{q^{\prime}}=\vec{q}\left(1-\beta q+\frac{\beta^{3}}{3} q^{2}\right)
$$

Thus,

$$
\epsilon(\vec{q})=1+\frac{\lambda^{2}}{q^{2}\left(1+\left(2 \beta^{2} / 3\right) q^{2}-2 \beta q\right)} .
$$

To calculate the total potential $\mathscr{U}(r)$, we preform the Fourier transform of $\mathcal{U}(\vec{q})$ :

$$
\mathcal{U}(\vec{r})=\frac{1}{(2 \pi)^{3}} \int \frac{d^{3} q}{1-\beta q+\beta^{2} q^{2}}\left(\frac{\mathcal{U}_{0}(\vec{q})}{\epsilon(\vec{q})}\right) e^{i \vec{q} \cdot \vec{r}} .
$$

As before we have

$$
\mathcal{U}_{0}\left(\overrightarrow{q^{\prime}}\right)=-\frac{4 \pi e}{q^{\prime 2}}
$$

where $q^{\prime}$ is given by (100). Therefore, taking only the terms that are first order in $\beta$, we find

$$
\mathcal{U}_{0}(\vec{q})=-\frac{4 \pi e}{q^{2}\left(1+\left(2 \beta^{2} / 3\right) q^{2}-2 \beta q\right)} .
$$




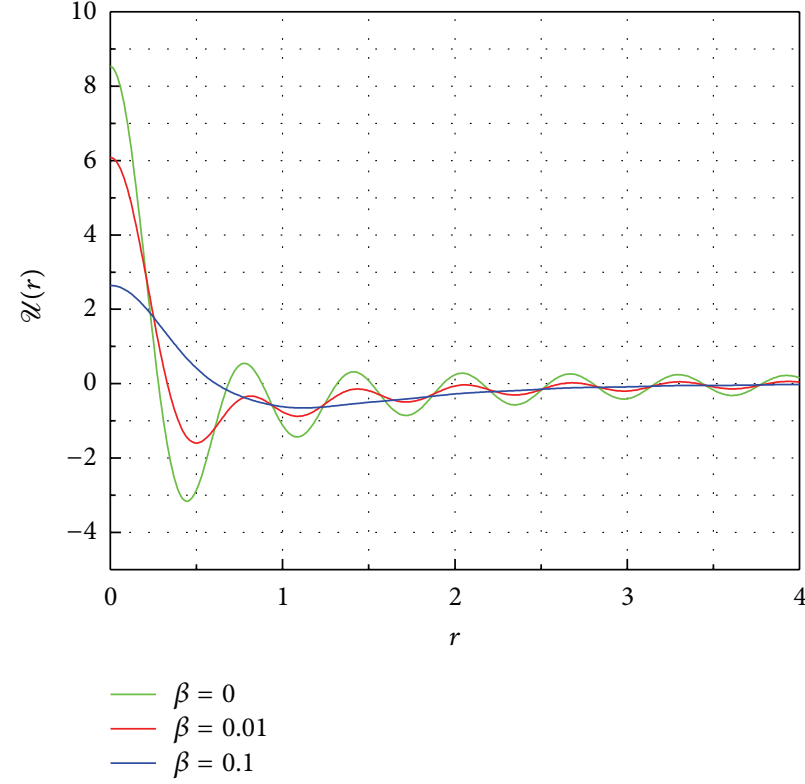

(a)

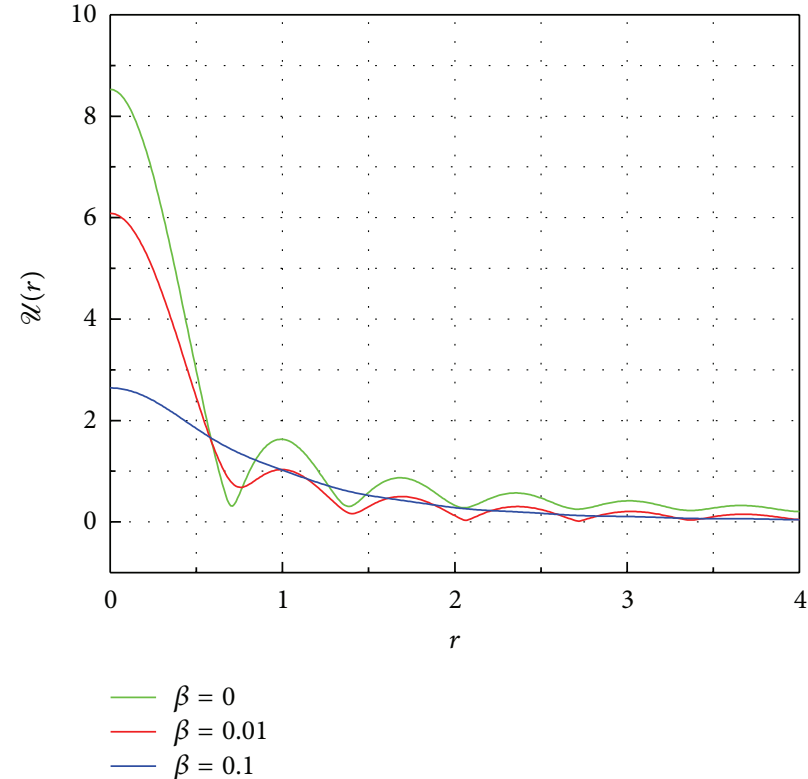

(b)

Figure 3: Real part (a) and norm (b) of $\mathscr{U}(r)$ for different values of $\beta$.

Now, the total potential can be written as

$$
\begin{aligned}
\mathscr{U}(r)=\frac{-4 \pi e}{(2 \pi)^{3}} & \int \frac{d^{3} q}{1-\beta q+\beta^{2} q^{2}} \\
& \times\left(\frac{1}{q^{2}\left(1+\left(2 \beta^{2} / 3\right) q^{2}-2 \beta q\right)+\lambda^{2}}\right) e^{i \vec{q} \cdot \vec{r}} .
\end{aligned}
$$

So we have

$$
\begin{aligned}
& \mathcal{U}(r) \\
& =\frac{-(4 \pi e)^{2} e}{(2 \pi)^{3}} \int \frac{q^{2} d q}{1-\beta q+\beta^{2} q^{2}} \\
& \quad \times\left(\frac{1}{q^{2}\left(1+\left(2 \beta^{2} / 3\right) q^{2}-2 \beta q\right)+\lambda^{2}}\right) e^{i \vec{q} \cdot \vec{r}} .
\end{aligned}
$$

Figure 3 shows the real part and also norm of $\mathcal{U}(r)$ for different values of $\beta$.

To estimate correlation energy between electrons defined as

$$
E_{\mathrm{CORR}}=\lim _{r \rightarrow 0}\left(-\frac{e}{2} V_{\mathrm{IND}}(r)\right)
$$

we take the Fourier transform of $V_{\mathrm{IND}}(\vec{q})$ to find

$$
\begin{aligned}
V_{\mathrm{IND}}(\vec{r})=\frac{1}{(2 \pi)^{3}} & \int \frac{d^{3} q}{1-\beta q+\beta^{2} q^{2}} \\
& \times \mathcal{U}_{0}(\vec{q})\left(\frac{1}{\epsilon(\vec{q})}-1\right) e^{i \vec{q} \cdot \vec{r}} ;
\end{aligned}
$$

thus,

$$
\begin{aligned}
E_{\mathrm{CORR}}= & \lim _{r \rightarrow 0} \frac{(4 \pi e)^{2}}{2(2 \pi)^{3}} \\
& \times \int \frac{q^{2} d q}{1-\beta q+\beta^{2} q^{2}} \\
& \times\left[\frac{1}{q^{2}\left(1+\left(2 \beta^{2} / 3\right) q^{2}-2 \beta q\right)+\lambda^{2}}\right. \\
& \left.\quad-\frac{1}{q^{2}\left(1+\left(2 \beta^{2} / 3\right) q^{2}-2 \beta q\right)}\right] e^{i \vec{q} \cdot \vec{r}} .
\end{aligned}
$$

Note that, due to the presence of the maximal momentum of the order of Planck momentum, this integral should be calculated for $q$ ranging from $-q_{\mathrm{pl}}$ to $+q_{\mathrm{pl}}$ that $q_{\mathrm{pl}}=p_{\mathrm{pl}} / \hbar$. Figure 4 shows the real part and also the norm of $E_{\text {CORR }}$ for different values of $\beta$.

Finally we note that it is possible to derive numerical bound on the value of the GUP parameter $\beta$ or the parameter $\beta_{0}$ defined as $\beta=\beta_{0} / M_{\mathrm{pl}} c$. As we have shown in [34], inspection of a bouncing particle in a gravitational field reveals the bound $\beta_{0}<10^{29}$. However, authors of [37] obtained the stronger bound of $\beta_{0}<10^{10}$ by treating the Lamb shift in hydrogen atom.

\section{Summary and Conclusions}

In this paper the one-dimensional quantum $N$-body problem has been studied in condensed matter physics within the framework of a modified Heisenberg algebra admitting a 


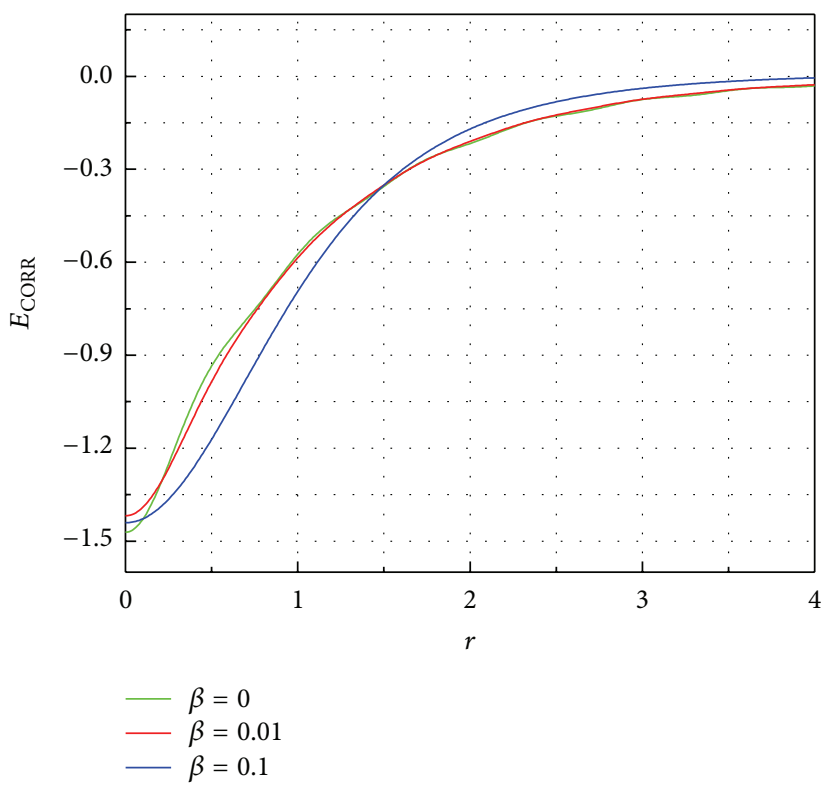

(a)

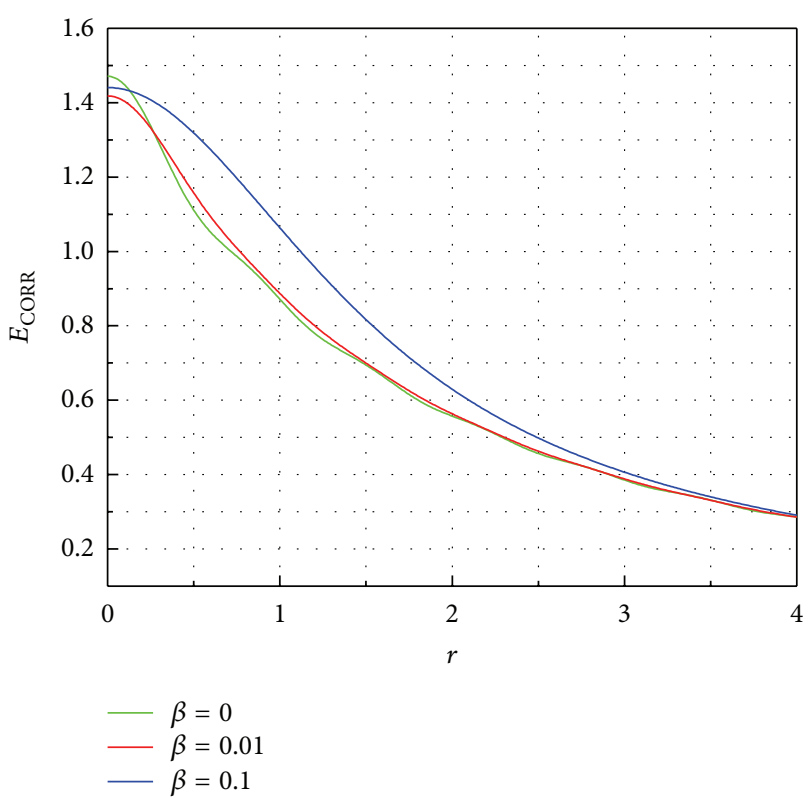

(b)

Figure 4: Real part (a) and norm (b) of $E_{\text {CORR }}$ for different values of $\beta$.

minimal measurable length and maximal momentum. We have introduced within some approximation the effective Hamiltonian describing a solid and the interaction potential between electrons in this system. The formalism developed has been explicitly applied to the case of Thomas-Fermi theory that is a basic theory in many-body interactions. The system under study is an electronic gas in equilibrium that is disturbed with a local electrostatic force. At the first step we investigated this system in the presence of a minimal length and obtained total potential and interaction potential between electrons and then correlation energy and generalized dielectric function in this setup. Then we generalized our study to the case where there are both minimal length and maximal momentum as natural cutoffs. We have shown that, in contrast to the undeformed space, in this deformed space there are some new contributions in total potential and correlation energy that are determined by the deformation parameter. As an important result, we have shown that corrections to the dielectric function in deformed space are wave number dependent. This feature may provide a clue to see these tiny effects in the lab in future experiments. In conclusion we would like to stress that the formulation proposed here could be very useful in studying properties of particles in many-body systems in condensed matter physics and their various associated observables in the context of the deformed Heisenberg algebra.

\section{Conflict of Interests}

The authors declare that there is no conflict of interests regarding the publication of this paper.

\section{Acknowledgment}

This work is financially supported by the Research Council of the Islamic Azad University, Sari Branch, Sari, Iran.

\section{References}

[1] G. Veneziano, "A stringy nature needs just two constants," Europhysics Letters, vol. 2, no. 3, article 199, 1986.

[2] D. Amati, M. Cialfaloni, and G. Veneziano, "Superstring collisions at planckian energies," Physics Letters B, vol. 197, no. 1-2, pp. 81-88, 1987.

[3] D. Amati, M. Cialfaloni, and G. Veneziano, "Can spacetime be probed below the string size?" Physics Letters B, vol. 216, no. 1-2, pp. 41-47, 1989.

[4] D. J. Gross and P. F. Mende, “The high-energy behavior of string scattering amplitudes," Physics Letters B, vol. 197, no. 1-2, pp.129134, 1987.

[5] K. Konishi, G. Paffuti, and P. Provero, "Minimum physical length and the generalized uncertainty principle in string theory," Physics Letters B, vol. 234, no. 3, pp. 276-284, 1990.

[6] R. Guida, K. Konishi, and P. Provero, "On the short distance behavior of string theories," Modern Physics Letters A, vol. 6, no. 16, pp. 1487-1504, 1991.

[7] M. Kato, "Particle theories with minimum observable length and open string theory," Physics Letters B, vol. 245, no. 1, pp. 4347, 1990.

[8] L. J. Garay, "Quantum gravity and minimal length," International Journal of Modern Physics A, vol. 10, no. 2, pp. 145-166, 1995.

[9] S. Capozziello, G. Lambiase, and G. Scarpetta, "Generalized uncertainty principle from quantum geometry," International Journal of Theoretical Physics, vol. 39, no. 1, pp. 15-22, 2000. 
[10] M. Maggiore, "A generalized uncertainty principle in quantum gravity," Physics Letters B, vol. 304, no. 1-2, pp. 65-69, 1993.

[11] M. Maggiore, "The algebraic structure of the generalized uncertainty principle," Physics Letters B, vol. 319, no. 1-3, pp. 83-86, 1993.

[12] M. Maggiore, "Quantum groups, gravity, and the generalized uncertainty principle," Physical Review D, vol. 49, no. 10, pp. 5182-5187, 1994.

[13] S. Hossenfelder, "Minimal length scale scenarios for quantum gravity," Living Reviews in Relativity, vol. 16, 2013.

[14] S. Hossenfelder, "Can we measure structures to a precision better than the Planck length?" Classical and Quantum Gravity, vol. 29, no. 11, Article ID 115011, 2012.

[15] F. Scardigli, "Generalized uncertainty principle in quantum gravity from micro-black hole gedanken experiment," Physics Letters B, vol. 452, no. 1-2, pp. 39-44, 1999.

[16] A. Kempf, G. Mangano, and R. B. Mann, "Hilbert space representation of the minimal length uncertainty relation," Physical Review D, vol. 52, no. 2, pp. 1108-1118, 1995.

[17] H. Hinrichsen and A. Kempf, "Maximal localization in the presence of minimal uncertainties in positions and in momenta," Journal of Mathematical Physics, vol. 37, no. 5, pp. 2121-2137, 1996.

[18] A. Kempf, "On quantum field theory with nonzero minimal uncertainties in positions and momenta," Journal of Mathematical Physics, vol. 38, no. 3, pp. 1347-1372, 1997.

[19] M. Bojowald and A. Kempf, "Generalized uncertainty principles and localization of a particle in discrete space," Physical Review D, vol. 86, no. 8, Article ID 085017, 15 pages, 2012.

[20] K. Nozari and A. Etemadi, "Minimal length, maximal momentum, and Hilbert space representation of quantum mechanics," Physical Review D, vol. 85, no. 10, Article ID 104029, 12 pages, 2012.

[21] R. J. Adler, "Six easy roads to the Planck scale," The American Journal of Physics, vol. 78, article 925, 2010.

[22] G. Amelino-Camelia, "Relativity in spacetimes with shortdistance structure governed by an observer-independent (Planckian) length scale," International Journal of Modern Physics D, vol. 11, no. 1, pp. 35-59, 2002.

[23] G. Amelino-Camelia, "Special treatment," Nature, vol. 418, no. 6893, pp. 34-35, 2002.

[24] G. Amelino-Camelia, "Doubly-special relativity: first results and key open problems," International Journal of Modern Physics D, vol. 11, no. 10, pp. 1643-1669, 2002.

[25] J. Kowalski-Glikman, "Introduction to doubly special relativity," in Planck Scale Effects in Astrophysics and Cosmology, vol. 669 of Lecture Notes in Physics, pp. 131-159, Springer, Berlin, Germany, 2005.

[26] G. Amelino-Camelia, G. Mandanici, A. Procaccini, and J. Kowalski-Glikman, "Phenomenology of doubly special relativity," International Journal of Modern Physics A, vol. 20, no. 26, pp. 6007-6037, 2005.

[27] K. Imilkowska and J. Kowalski-Glikman, "Doubly special relativity as a limit of gravity," in Special Relativity, vol. 702 of Lecture Notes in Physics, pp. 279-298, Springer, Berlin, Germany, 2006.

[28] J. Magueijo and L. Smolin, "Lorentz invariance with an invariant energy scale," Physical Review Letters, vol. 88, no. 19, Article ID 190403, 4 pages, 2002.

[29] J. Magueijo and L. Smolin, "Generalized Lorentz invariance with an invariant energy scale," Physical Review D, vol. 67, no. 4, Article ID 044017, 12 pages, 2003.
[30] J. Magueijo and L. Smolin, "String theories with deformed energy-momentum relations, and a possible nontachyonic bosonic string," Physical Review D, vol. 71, no. 2, Article ID 026010, p. 6, 2005.

[31] J. L. Cortés and J. Gamboa, "Quantum uncertainty in doubly special relativity", Physical Review D, vol. 71, no. 6, Article ID 065015, p. 4, 2005.

[32] A. F. Ali, S. Das, and E. C. Vagenas, "Discreteness of space from the generalized uncertainty principle," Physics Letters B, vol. 678, no. 5, pp. 497-499, 2009.

[33] S. Das, E. C. Vagenas, and A. Farag Ali, "Discreteness of space from GUP II: relativistic wave equations," Physics Letters B, vol. 690, pp. 407-412, 2010.

[34] P. Pedram, K. Nozari, and S. H. Taheri, "The effects of minimal length and maximal momentum on the transition rate of ultra cold neutrons in gravitational field," Journal of High Energy Physics, vol. 2011, article 93, 2011.

[35] J. C. Inkson, Many-Body Theory of Solids, Plenum Press, New York, NY, USA, 1984.

[36] S. Das and E. C. Vagenas, "Universality of quantum gravity corrections," Physical Review Letters, vol. 101, Article ID 221301, 4 pages, 2008.

[37] A. Farag Ali, S. Das, and E. C. Vagenas, "Proposal for testing quantum gravity in the lab," Physical Review D, vol. 84, Article ID 044013, 2011. 

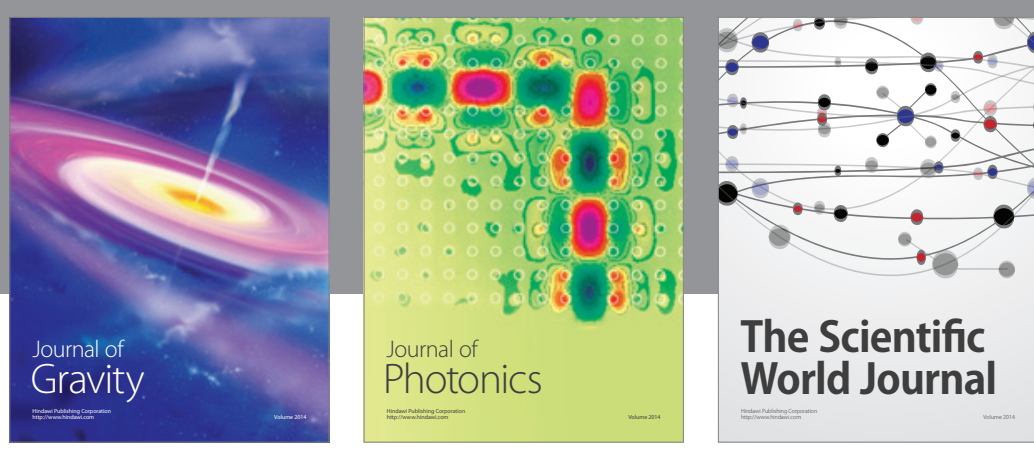

The Scientific World Journal
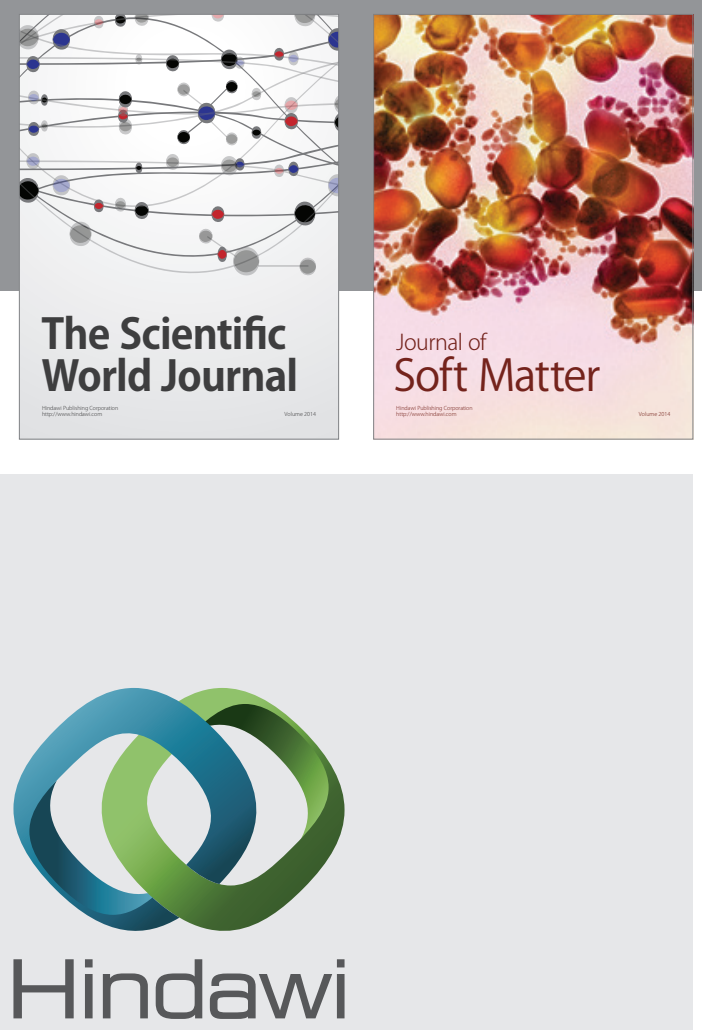

Submit your manuscripts at

http://www.hindawi.com

nternational Journal of

Statistical Mechanics
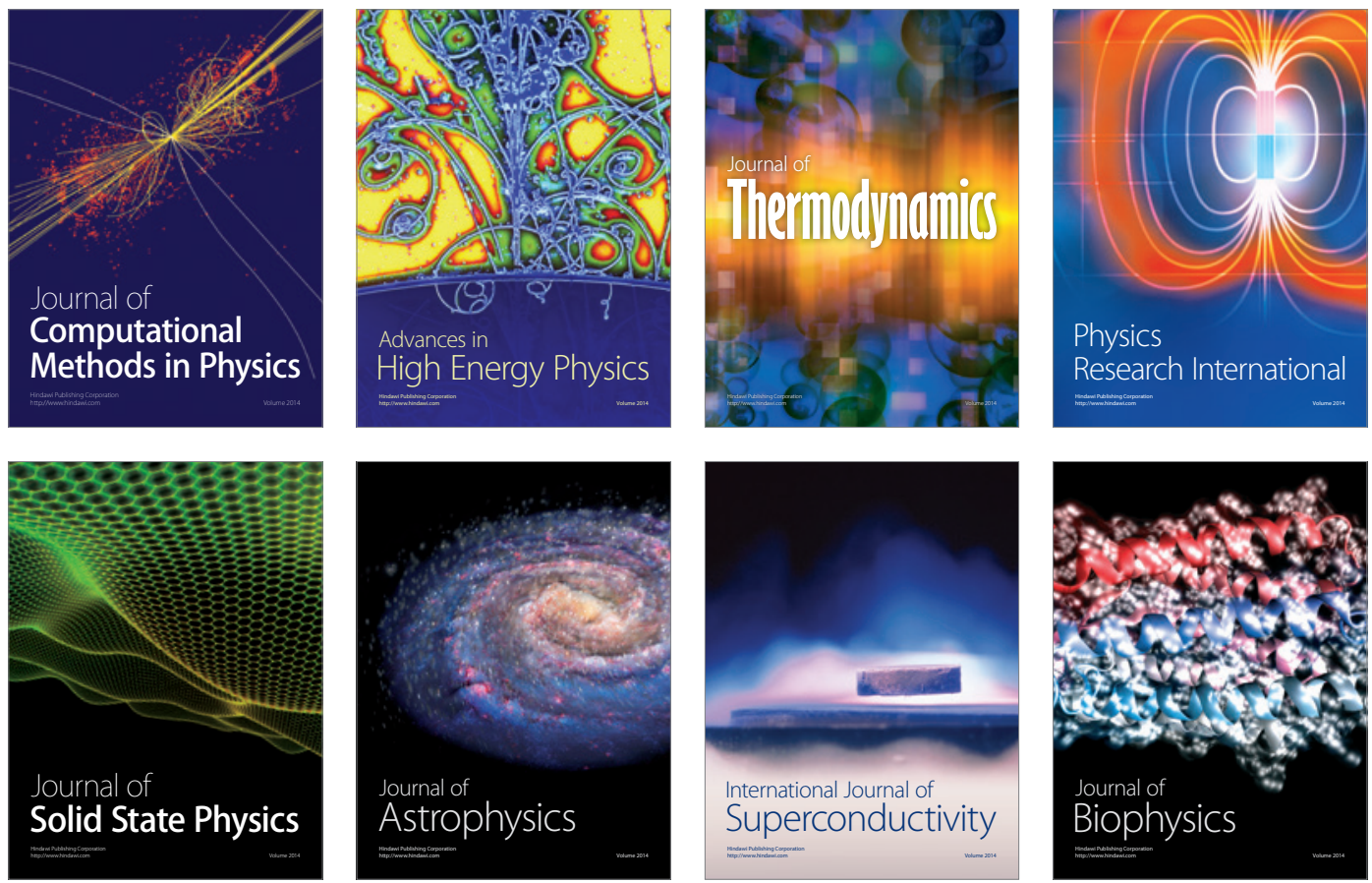
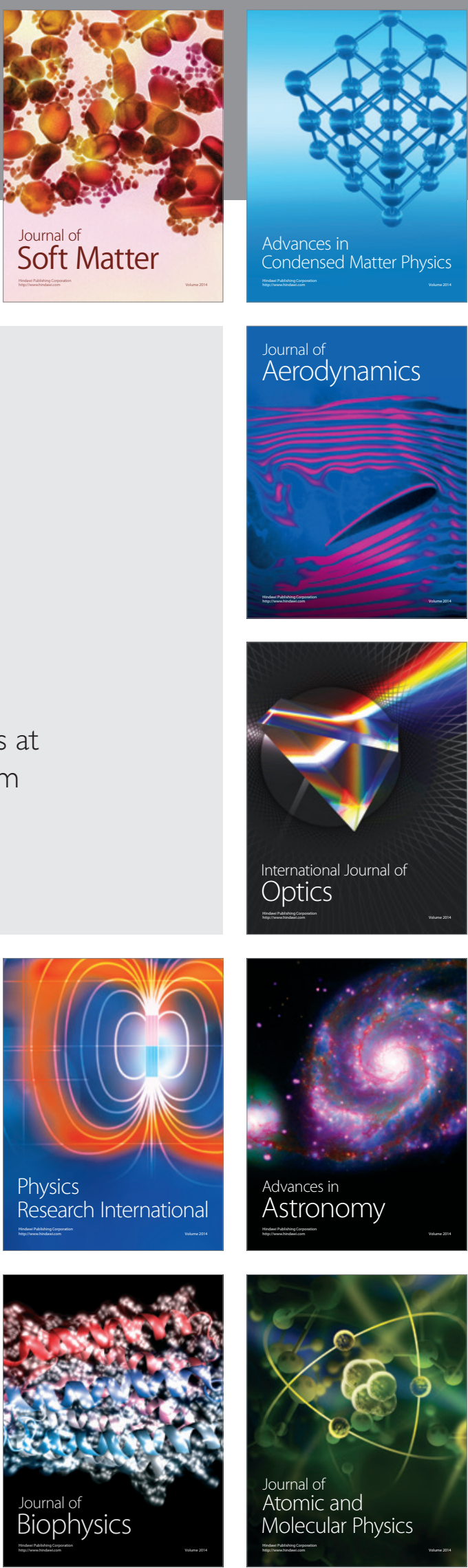